\title{
Real-world experience with new migraine treatments in Brazil Preliminary results
}

\author{
Abouch Valenty Krymchantowski ii \\ Ana Gabriela Ferreira Krymchantowski \\ Carla da Cunha Jevoux
}

Headache Center of Rio, Rio de Janeiro, RJ, Brazil.

\section{$凶$}

Abouch V Krymchantowski Headache Center of Rio, Rua Siqueira Campos 43/1002 Copacabana 22031.071, Rio

de Janeiro, RJ, Brazil. abouchkry@uol.com.br

This article was edited by: Marcelo Moraes Valença Juliana Ramos de Andrade

\section{Keywords:}

Migraine

Treatment

CGRP

Upcoming

Medications

Monoclonal antibodies

\begin{abstract}
Introduction Migraine is a highly prevalent disorder. Current treatments are far from desired regarding efficacy and tolerability. Recent knowledge has been indicating targets whose antagonism may improve outcomes. Blocking CGRP or its receptor with monoclonal antibodies $(\mathrm{mAb})$ can interfere with migraine mechanisms and decrease the frequency of attacks. Erenumab, galcanezumab, fremanezumab and epitnezumab were recently approved for migraine treatment and the first three are available in Brazil. Although the figures of efficacy were not astonishing, tolerability and higher adherence were demonstrated. However, real-world experience is limited in Brazil, since these new therapies have been used for only a year.
\end{abstract}

Objective We present a summary of pivotal studies with the four mAb and preliminary results of this short-term option for migraine in real-world Brazilian patients.

Methods One hundred twelve episodic or chronic migraineurs received the prescription of a mAb during the last 12 months in a tertiary center. This initial study was performed with 83 patients who should have taken, at the time of this writing, at least three-monthly doses of a mAb, which was chosen by a neurologist with full time dedication to headache medicine.

Results Sixty-four women (77.1\%) and 19 men (22.9\%), with episodic ( $n=49 ; 59 \%$ ) or chronic migraine $(n=34 ; 41 \%)$, mean age of 43.6 years and mean headache history of 26.2 years were retrospectively studied. Baseline frequency was 14.8 headache days/month in the three months prior to the $\mathrm{mAb}$ use. Erenumab was prescribed to 40 (48.2\%) patients; galcanezumab was given to $37(44.6 \%)$ patients and fremanezumab was prescribed to 6 $(7.2 \%)$ migraineurs. The mean headache frequency among the 77 patients who returned was reduced to 5.6 headache days/month in the following three months. Considering the headache frequency reduction of $\geq 50 \%, 44$ (57\%) migraine sufferers achieved a meaningful decrease. Mild adverse events were presented by $24.6 \%$ of the patients.

Conclusion The authors present the first Brazilian experience in real-world patients using different $\mathrm{mAbs}$ in migraineurs' preventive treatment. 


\section{Introduction}

M igraine is a highly prevalent neurological disorder. ${ }^{1}$ It causes substantial burden, but treatments, especially preventive, were chosen by serendipity. ${ }^{1,2}$ Despite effectiveness, at least partial, of numerous drugs, outcomes were distant from the expected by patients and treating physicians. Poor adherence due to tolerability issues and limited efficacy were commonly seen. ${ }^{2,3}$

The knowledge about migraine pathophysiology is still uncertain. 4,5 Peptides involved in the complex process of migraine attacks, such as calcitonin gene-related peptide (CGRP), have been implicated and identified as targets for migraine therapies. ${ }^{4,5}$

CGRP is a 37-amino acid peptide densely present in the hypothalamus, thalamus and cerebellum as well as in sensory fibers and neurons involved in pain transmission at dorsal root ganglia and trigeminal ganglia. In addition, CGRP is also present in the peripheral nervous system. ${ }^{4,5}$

The binding receptor of CGRP, described as a G-protein coupled receptor, has two subunits composed by a calcitonin receptor-like subunit (CLR) and an activity-modifying protein 1 subunit known as RAMP1. ${ }^{6}$ The critical role of this peptide in migraine has been emphasized by the fact that its serum levels are elevated in episodic migraine attacks, the intravenous infusion of CGRP triggers attacks in migraineurs and CGRP concentrations in jugular veins blood rise during headache attacks of migraine. Additionally, CGRP serum level decreases with symptomatic relief of the headache. ${ }^{6,7}$

There are different antagonists of the CGRP or its receptor. The last 20 years of research have identified potential agents either for the acute treatment as well as for the prevention of migraine. ${ }^{4,5,7,8,9}$ Specifically for migraine prevention, biological options such as monoclonal antibodies anti-CGRP or its receptor started to be developed and four were recently approved by the FDA and Anvisa. ${ }^{7,8,10}$

\section{New therapies}

Four monoclonal antibodies ( $\mathrm{mAb}$ ) anti-CGRP were approved for migraine prevention during the last three years. Commercially, there are three already available in Brazil. Erenumab is the only monoclonal antibody against the CGRP receptor whereas galcanezumab, fremanezumab and epitnezumab act on the peptide CGRP itself. ${ }^{8}$ The latter is likely to be launched in Brazil soon.

There is a body of excitement regarding these new treatment options. All four mAb have long half-lifes, restricted tissue penetrance and highly selective affinity for the CGRP itself or its receptor. Therefore, it has become the center of the upcoming arsenal, although CGRP nerve endings are extraluminal in most tissues, which may impair and limit its efficacy. ${ }^{4,5}$

Two phase 3 pivotal registration trials were recently published with erenumab. ${ }^{11,12}$ The STRIVE (Study to Evaluate the Efficacy and Safety of Erenumab in Migraine Prevention) trial evaluated subjects presenting 4-14 migraine days/month during 6 months in three arms. Among the 955 randomized patients, 319 received $140 \mathrm{mg}, 317$ received $70 \mathrm{mg}$ and 319 received placebo in monthly subcutaneous injections. In the primary endpoint of reduction in mean migraine days per month, compared to the previous 3-month baseline period, the 140 $\mathrm{mg}$ group had a higher reduction of 3.7 days. The $70 \mathrm{mg}$ dose group reduced headache frequency by 3.2 days and placebo group of 1.8 days $(p<.001$ for each dose vs. placebo). In the secondary endpoints of $\geq 50 \%$ responder rate in mean monthly migraine days, days with use of migraine-specific medications and changes of score for everyday activities and general physical impairment, both doses were significantly better than placebo. ${ }^{11}$

The second phase 3 pivotal study included 570 patients who were treated for 3 months either with the 70 mg-dose or placebo. The change in mean monthly migraine days from weeks 9-12 compared to the baseline was also the primary endpoint. Erenumab lead to a mean reduction of 2.9 days vs. 1.8 days of the placebo group $(p<.001)$.

In both trials, no serious adverse events were observed, but injection-site reactions or local pain were presented by $3.2 \%$ to $6 \%$ of those having used erenumab. Mild arthralgia and bothersome constipation were also observed with erenumab in the STRIVE study. ${ }^{12}$

Fremanezumab was the second mAb approved by the FDA, but the third launched in Brazil. The trials involved episodic migraine sufferers, high-frequency episodic migraineurs and chronic migraineurs. In high-frequency migraineurs, different doses were used in patients with 8-14 headache days during a 4-week baseline period, who were randomized to receive either $675 \mathrm{mg}$ (followed by two placebo doses), $225 \mathrm{mg}$ monthly doses or placebo every 4 weeks during the study timeframe of 12 weeks. ${ }^{13}$ Efficacy endpoint was measured by the change in number of migraine days during the weeks 9-12 compared to the frequency baseline. Both doses promoted significantly greater reductions compared to placebo. ${ }^{13}$

The monthly doses of $225 \mathrm{mg}$ resulted in a reduction of -6.27 vs. -3.46 of the placebo and $675 \mathrm{mg}$ resulted in a reduction of 6.09 vs. 3.46 days of the placebo $(p<.0001) .{ }^{13}$

In a pivotal phase 3 registration trial, the HALO study, the mean change in migraine days, was compared among 875 patients (742 women; 133 men) with mean age of 41.8 years. The patients who received $225 \mathrm{mg}$ per month (3 doses) had a reduction from 8.9 days to 4.9 days vs. 9.2 days to 5.3 days in patients who received a single dose of $675 \mathrm{mg}$. The 
placebo group reduced migraine days from 9.1 days to 6.5 days $(p<.001) \cdot{ }^{14}$

Tolerability issues were not different between placebo and treatment groups and erythema and induration at injection sites ( $n=3$ and 2, respectively) were the most frequent adverse events presented by the studied patients. ${ }^{14}$

Fremanezumab was also evaluated for the prevention of chronic migraine ${ }^{15}$ in patients randomized to receive the initial loading dose of $675 \mathrm{mg}$ followed by two monthly doses of $225 \mathrm{mg}$ or three-monthly doses of $900 \mathrm{mg}$ or placebo. The mean reduction in headache hours of any intensity during the weeks 9-12 was significantly greater compared to placebo either for the highest dose as for the $675 \mathrm{mg} / 225 \mathrm{mg}$ and $225 \mathrm{mg}$ subjects. The patients who received three doses of $900 \mathrm{mg}$ presented less 67.5 hours vs. -37.1 hours ( $p=.0057)$. The difference between the patients who received $675 \mathrm{mg} / 225 \mathrm{mg}$ and $225 \mathrm{mg}$ was -59.8 hours vs. 37.1 hours ( $p=.038$ ). It is noteworthy that the mean number of headache days per month at baseline was 16 for all groups, which may not allow definitive comparisons with daily or near-daily headache sufferers as commonly seen in tertiary centers. ${ }^{15}$

Another phase 3 pivotal trial of fremanezumab for the prevention of chronic migraine enrolled 1,130 patients randomized to receive $675 \mathrm{mg}$ as loading dose followed by two doses of placebo at weeks 4 and 8 (376 patients) or $675 \mathrm{mg}$ as loading dose at baseline and $225 \mathrm{mg}$ at weeks 4 and 8 (379 patients) or placebo (375 patients). ${ }^{16}$ The change in headache days in which migraine-specific medications were used was one of the primary endpoints. ${ }^{16}$

Baseline frequency of migraine days were 16.4 days for the placebo group, 16.2 days for the quarterly dose group and 16 days for the monthly dose group. Fremanezumab every month resulted in migraine days reduction of $4.6 \pm 0.3$. Fremanezumab quarterly showed a reduction of $4.3 \pm 0.3$ days and the placebo patients revealed a $2.5 \pm 0.3$ reduction in headache days ( $p<.001$ for both comparisons with placebo).

Galcanezumab was studied in two phase 3 trials (EVOLVE 1 and EVOLVE2) for the preventive treatment of migraine. ${ }^{17,18}$ The EVOLVE 1 trial was a randomized, double-blind, placebo-controlled comparison between galcanezumab $120 \mathrm{mg}$, $240 \mathrm{mg}$ or placebo. The patients received monthly doses for 6 months. Patients (18-65 years) had a minimum of 1-year history of migraine and 4-14 migraine headache days per month. A total of 858 patients were included in the intention-to-treat population. Contrarily to other mAbs studies, no other preventive medications were allowed during the study. ${ }^{17}$

Primary outcome was the mean change in number of monthly migraine headache days and secondary endpoints were a frequency reduction of at least $50 \%$, of at least $75 \%$, and of $100 \%$. In addition, migraine headache days with acute medication utilization was also compared between goups. ${ }^{17}$
The primary endpoint was achieved for both galcanezumab doses and the active treatment significantly reduced monthly migraine headache days by 4.7 days $(120 \mathrm{mg})$ and by 4.6 days (240 mg) compared with placebo (2.8 days) (for both $\mathrm{p}<.001$ ). Secondary endpoints were significantly superior to placebo either for galcanezumab $120 \mathrm{mg}$ and $240 \mathrm{mg} .{ }^{17}$

The EVOLVE 2 involved 915 intention-to-treat patients. They either received monthly subcutaneous injections of placebo $(n=461)$, galcanezumab $120 \mathrm{mg}(n=231)$ or $240 \mathrm{mg}$ $(n=223)$ during 6 months. ${ }^{18}$ The primary endpoint was the mean change in monthly migraine headache days and other key secondary endpoints were response rates of $\geq 50 \%, \geq 75 \%$, and $100 \%$ in addition to reduction of monthly migraine headache days with acute medication use. ${ }^{18}$

At baseline, $66.9 \%$ of patients had $\geq 8$ migraine headache days/month and most of the subjects (65.5\%) had prior experience with migraine preventive medications. Interestingly, $14.3 \%$ had previously failed to two or more pharmacological agents. ${ }^{18}$ Monthly migraine days were reduced by 4.3 and by 4.2 days with galcanezumab $120 \mathrm{mg}$ and $240 \mathrm{mg}$, while placebo reduced headache days by 2.3 and group differences $(95 \% \mathrm{Cls})$ vs. placebo were $2.0(-2.6,-1.5)$ and $1.9(-2.4$, -1.4), respectively. Both doses were superior to placebo for all key secondary endpoints and the occurrence of injection site reactions was the most common adverse event. ${ }^{18}$

Eptinezumab is the only $\mathrm{mAb}$ in intravenous injections and was the last approved for commercial use in the United States and Brazil. PROMISE 1 and 2 were the two phase 3 pivotal trials for migraine prevention. It stands for "Prevention of Migraine via Intravenous eptinezumab Safety and Efficacy". The first trial evaluated 888 migraineurs with 4-14 migraine days per 30days, who were randomized to receive quarterly intravenous infusions of either eptinezumab doses of $30 \mathrm{mg}, 100 \mathrm{mg}$ or $300 \mathrm{mg}$ or placebo. The primary endpoint was the reduction in migraine days over weeks 1-12. Baseline frequency among groups was similar reaching 8.4 to 8.7 days. The $300 \mathrm{mg}$ dose reduced migraine days by -4.3 compared to -3.2 of the placebo group and -4 and -3.9 , respectively for $30 \mathrm{mg}$ and $100 \mathrm{mg}$ doses ( $p<.0001$ for $300 \mathrm{mg}$ vs. placebo; $\mathrm{p}=.0046$ for $30 \mathrm{mg}$ vs. placebo and $\mathrm{p}=.0182$ for $100 \mathrm{mg}$ vs. placebo)..$^{19}$

Responder rates of $\geq 75 \%$ and $\geq 50 \%$ were secondary end-points also evaluated and compared between groups for the weeks $1-4$ and 1-12. As for the weeks 1-4, 31.5\% of the $300 \mathrm{mg}$ patients vs. $20.3 \%$ of the placebo group revealed a responder rate of higher than $75 \%(p=.0066)$. Additionally, $51 \%$ of those having received $300 \mathrm{mg}$ achieved $\geq 75 \%$ reduction in days of migraine after the $3^{\text {rd }}$ and $4^{\text {th }}$ infusions. ${ }^{19}$

Tolerability profile was similar among groups. Upper respiratory infection occurred in $11 \%$ of the $30-\mathrm{mg}, 10 \%$ in the $100-\mathrm{mg}, 10 \%$ in $300-\mathrm{mg}$ and $7 \%$ of the placebo groups. 
The PROMISE-2 study was designed to evaluate the efficacy in the prevention of chronic migraine. ${ }^{20}$ The patients had $\geq 15$ to 26 headache days per month with at least 8 migraine days. They either received placebo or epitnezumab in the doses of $100 \mathrm{mg}$ and $300 \mathrm{mg}$ in intravenous quarterly injections. Baseline migraine frequency was 16.2 for the placebo group and 16.1 days for the active antibody groups. Changes in mean migraine days per month during weeks 1-12 was the primary endpoint. Reductions were, respectively, -8.2 days for 300-mg group, -7.7 for the 100-mg group and -5.6 days for the placebo group ( $p<.0001$ for both doses vs. placebo). ${ }^{20}$

\section{Real-world experience in Brazil}

In the Headache Center of Rio de Janeiro, during the period February 2020 to February 2021, anti-CGRP mAbs were prescribed to 112 migraine patients. The results and outcomes are being collected and tabulated. As of the writing of this initial review, we have data on 83 patients to whom a mAb prescribed before November the 1st, 2020 (Table 1). The monoclonal antibody prescribed was chosen by the treating professionals, who are neurologists with especial dedication to Headache Medicine and clinical experience of at least 25 years working in tertiary centers.

Table 1. Patients demographics and outcomes

\begin{tabular}{|c|c|c|c|}
\hline & $n$ & $\%$ & Mean \\
\hline Women & 64 & 77.1 & \\
\hline Men & 19 & 22.9 & \\
\hline Ages (years) & $21-76$ & & 43.6 \\
\hline Episodic migraine & 49 & 59 & \\
\hline Chronic migraine & 34 & 41 & \\
\hline Medication-overuse headache & 31 & 37.3 & \\
\hline Psychiatric comorbidities* & 28 & 33.7 & \\
\hline Preventive pharmacological medications & 1.4 & & 2.2 \\
\hline Headache history (years) & $6-60$ & & 26.2 \\
\hline Monthly headache days prior to $\mathrm{mAb}^{\dagger}$ & & & 14.8 \\
\hline 3 months & 14.9 & & \\
\hline 2 months & 14.5 & & \\
\hline 1 month & 15 & & \\
\hline Erenumab & 40 & 48.2 & \\
\hline Galcanezumab & 37 & 44.6 & \\
\hline Fremanezumab & 6 & 7.2 & \\
\hline Monthly headache days after the $\mathrm{mAb}^{\dagger}$ & & & 5.6 \\
\hline 1 month after the mAb & 4 & & \\
\hline 2 months after the mAb & 5 & & \\
\hline 3 months after the $\mathrm{mAb}$ & 8 & & \\
\hline Patients with $\geq 50 \%$ frequency headache reduction & 44 & 57 & \\
\hline Adverse events ${ }^{\ddagger}$ & 19 & 24.6 & \\
\hline
\end{tabular}

*Anxiety, depression, or personality disturbances; ${ }^{\dagger}$ monoclonal antibody; *constipation, vertigo, nasopharyngitis, and injection site irritation.

Sixty-four female patients (77.1\%) and 19 men (22.9\%), ages 21.76 years (mean 43.6 ) with 6 to 60 years of headache history (mean 26.2) were retrospectively studied. They belong to a subset of 112 consecutive migraineurs to which a mAb was prescribed with a minimum of three-monthly doses within the timeframe of this study. Among the subjects, 49 (59\%) had episodic and 34 (41\%) had chronic migraine. Thirty-one chronic migraineurs $(37.3 \%)$ had also the diagnosis of medication-overuse headache.

The mean number of monthly headache days three months before the use of the first mAb dose was 14.9. The headache frequency two and one month before the use of the first $\mathrm{mAb}$ dose was, respectively, 14.5 and 15 headache days. Collectively, the mean number of monthly headaches days was 14.8 in the three months prior to the mAb use. We did not compare yet the mean headache frequency among episodic and chronic migraineurs, but this is one of the objectives of future studies with this population of patients.

All patients were being treated in our center for at least three months and the number of preventive pharmacological agents varied from 1 to 4 (mean 2.2) at the time of choosing the monoclonal antibody. None of the patients had alterations in medication dosages during the previous three months prior to the mAb use. Interestingly, 28 (33.7\%) patients had psychiatric comorbidities varying from anxiety, depression, or personality disorders at the time of receiving the new therapy.

The choice of the specific monoclonal antibody was performed due to personal reasons since no head-to-head studies are available comparing the three mAbs efficacy and tolerability. Prices, delivery systems, attitude of the pharmaceutical industry towards rightfulness and recognition of professional as well as ethics value of the prescribers, did fundament the choices.

Erenumab in monthly doses of $70 \mathrm{mg}$ was prescribed to 40 (48.2\%) patients; galcanezumab in a $240 \mathrm{mg}$ loading dose, followed by monthly doses of $120 \mathrm{mg}$, was given to 37 $(44.6 \%)$ patients, whereas fremanezumab in monthly doses of $225 \mathrm{mg}$ was prescribed to $6(7.2 \%)$ migraineurs. The treatment duration, whether limited to the initial three months or longer, was decided according to outcomes and patient's satisfaction evaluated in consultations carried out three months following the use of the first mAb dose. Respectively, three patients from the erenumab and three from the galcanezumab groups did not return to follow up.

The mean headache frequency among the 77 patients who returned was, respectively, four, five and eight monthly headache days after one, two and three months following the $\mathrm{mAb}$ use, which results in a mean headache frequency of 5.6 days/month. Considering the endpoint of headache frequency reduction of $50 \%$ or higher, $44(57 \%)$ migraine sufferers achieved a meaningful decrease in headache days.

No comparisons were carried out so far regarding the rate of headache decrease among specific monoclonal antibodies type as well as between groups of patients taking erenumab, galcanezumab and fremanezumab with regard to the presence of episodic migraine, chronic migraine, medication-o- 
veruse headache and the presence of psychiatric comorbidities, but the authors believe that it is crucial to evaluate better the trea-tment performance and expect to do so when the data of more patients are to become available. In addition, we still do not know whether the lack of adherence varies according to the mAb type.

\section{Comments}

This preliminary report lacks crucial information. To study a subpopulation of migraineurs with mixed presentations and comorbidities is not the ideal design. Additionally, one might argue on whether the patients receiving one or other $\mathrm{mAb}$ were similar in baseline demographics since the average headache frequency was calculated as mean for the whole group. However, we aimed at presenting the first Brazilian experience in real-world patients. Although patients from tertiary centers cannot be compared with primary care sufferers or treatment naïve subjects regarding previous treatment experiences, use of preventive therapies, knowledge about the biological nature of migraine and even treatment expectations, the authors believe that reports of initial real-world experiences with this recently available way of approaching migraine, could represent hope and guidance for different instances of treatment and doubtful physicians not practicing headache medicine on daily basis. If the $\mathrm{mAb}$ prices are to be considered as a main stain for deciding the treatment, it becomes even worse in Brazil.

The higher percentage of patients presenting adverse events is also noteworthy in this study. Although no serious adverse were observed, constipation and injection site erythema were the most common complains; we also saw two patients with vertigo, two with insomnia and one with facial edema. A higher number of patients with adverse reactions after using a mAb was also described by Kanaa et al. ${ }^{21}$, who demonstrated nearly half of the patients with constipation or other mild symptoms as well as safety regarding cardiovascular effects. ${ }^{21,22}$

More information and outcomes must be obtained so far and questions regarding when and why a monoclonal anti-CGRP antibody should be prescribed, whether it works better in patients already being treated with traditional pharmacological agents or starting a migraine treatment without previous use of medications is warranted and is on the way for real Brazilian sufferers.

Conflict interest: There is no conflict of interest.

Funding:The authors received no specific funding for this work. Author contribuition: The authors contributed with equal participation in the elaboration of the article.

Abouch V Krymchantowski

https://orcid.org/0000-0001-8164-3507

Ana Gabriela Ferreira Krymchantowski

https://orcid.org/0000-0002-8453-2068

Carla da Cunha Jevoux

https://orcid.org/0000-0003-4344-1028

\section{References}

1. GBD 2016 Disease and Injury Incidence and Prevalence Collaborators. Global, regional, and national incidence, prevalence, and years lived with disability for 328 diseases and injuries for 195 countries, 1990-2016: a systematic analysis for the Global Burden of Disease Study 2016. Lancet 2017390:1211-1259.

2. Estemalik E, Tepper S. Preventive treatment in migraine and the new US guidelines. Neuropsychiatr Dis Treat 2013;9:709-720.

3. González-Hernández A, Marichal-Cancino BA, MaassenVanDenBrink A, Villalón CM. Side effects associated with current and prospective antimigraine pharmacotherapies. Expert Opin Drug Metabol Toxicol 2018; 14:2541.

4. Tso AR, Goadsby PJ. Anti-CGRP monoclonal antibodies: the next era of migraine prevention? Curr Treat Options Neurol 2017; 19:27

5. Messina R, Goadsby PJ. CGRP - a target for acute therapy in migraine: Clinical data. Cephalalgia 2018 Jan 1:333102418768095.

6. Mclatchie LM, Fraser NJ, Main MJ, Wise A, Brown J, Thompson $\mathrm{N}$, et al. RAMPs regulate the transport and ligand specificity of the calcitonin-receptor-like receptor. Nature 1998;393:333-339.

7. Edvinsson L, Ho TW. CGRP receptor antagonism and migraine. Neurotherapeutics 2010;7:164-175.

8. Tepper SJ. History and Review of anti-Calcitonin Gene-Related Peptide (CGRP) Therapies: From Translational Research to Treatment. Headache 2018; Sep 22. doi: 10.1111/head. 13379. [Epub ahead of print] Review.

9. Ho TW, Connor KM, Zhang Y, Pearlman E, Koppenhaver J, et al. Randomized controlled trial of the CGRP receptor antagonist telcagepant for migraine prevention. Neurology. 2014;83:958-966.

10. Loder EW, Robbins MS. Monoclonal antibodies for migraine prevention. Progress, but not a panacea. JAMA 2018;319:1985-1987.

11. Goadsby PJ, Reuter U, Hallström Y, et al. A controlled trial of erenumab for episodic migraine. $N$ Engl J Med. 2017;377:2123-2132.

12. Dodick DW, Ashina M, Brandes JL, Kudrow D, Lanteri-Minet M, Osipova V, Palmer K, Picard H, Mikol DD, Lenz RA. ARISE: A Phase 3 randomized trial of erenumab for episodic migraine. Cephalalgia. 2018;38:1026-1037

13. Bigal ME, Dodick DW, Rapoport A, Silberstein SD, Ma Y, Yang R et al. Safety, tolerability, and efficacy of TEV-48125 for preventive treatment of high- frequency episodic migraine: a multicenter, randomized, double-blind, placebo-controlled, phase $2 b$ study. Lancet Neurol 2015;14:1081-90.

14. Dodick DW, Silberstein SD, Bigal ME, Yeung PP, Goadsby PJ, Blanckenbiller T, Grozinski-Wolff M, Yang R, Ma $Y$, Aycardi E. Effect of Fremanezumab compared with placebo for prevention of episodic migraine: A randomized controlled trial. JAMA 2018;319:1999-2008.

15. Bigal ME, Edvinsson L, Rapoport A, Lipton RB, Spierings E, Diener H-C et al. Safety, tolerability, and efficacy of TEV-48125 for preventive treatment of chronic migraine: a multicentre, randomized, double-blind, placebo-controlled, phase 2b study. Lancet Neurol 2015; 14:1091. 100 
16. Silberstein SD, Dodick DW, Bigal ME, Yeung PP, Goadsby PJ, Blankenbiller T, Grozinski-Wolff $M$, Yang R, Ma Y, Aycardi E. Fremanezumab for the preventive treatment of chronic migraine. The New England Journal of Medicine. 2017;377:2113-22.

17. Stauffer VL, Dodick DW, Zhang Q, Carter JN, Ailani J, Conley RR. Evaluation of Galcanezumab for the Prevention of Episodic Migraine: The EVOLVE-1 Randomized Clinical Trial. JAMA Neurol. 2018:1212. (Epub ahead of print)

18. kljarevski V, Matharu M, Millen BA, Ossipov MH, Kim B-K, Yang JY. Efficacy and safety of galcanezumab for the prevention of episodic migraine: Results of the EVOLVE-2 Phase 3 randomized controlled clinical trial Cephalalgia 2018:38:1442-1454.

19. Dodick DW, Goadsby PJ, Silberstein SD, Lipton RB, Olesen J, Ashina M, Wilks K, Kudrow D, Kroll R, Kohrman B, Bargar R, Hirman J, Smith J; ALD403 study investigators. Safety and efficacy of ALD403, an antibody to calcitonin gene-related peptide, for the prevention of frequent episodic migraine: a randomised, double-blind, placebo-controlled, ex ploratory phase 2 trial. Lancet Neurol. 2014;13:1 100-1 107.

20. Saper JR, Lipton RB, Kudrow DB, et al. A Phase 3, Randomized, Double-blind, Placebo-Controlled Study to Evaluate the Effectiveness and Safety of Eptinezumab in Frequent Episodic Migraine Prevention: Primary Results of the PROMISE 1 (PRevention Of Migraine via Intravenous eptinezumab Safety and Efficacy 1) Trial. PO-01-194. Cephalalgia 2017;37:337. (abstract).

21. Kanaan S, Hettie G, Loder E, Burch R. Real-world effectiveness and tolerability of erenumab: A retrospective cohort study. Cephalalgia 2020;40:151 1-1522.

22. Depre C, Antalik L, Starling A, Koren M, Eisele O, Lenz RA, Mikol DD. A Randomized, Double-Blind, Placebo-Controlled Study to Evaluate the Effect of Erenumab on Exercise Time During a Treadmill Test in Patients With Stable Angina. Headache. 2018;58:715-723. 